\title{
Téoros
}

Revue de recherche en tourisme

\section{La science du tourisme dans le discours des acteurs internationaux \\ Débat ou polémique?}

\section{Mimoun Hillali}

Volume 27, numéro 1, printemps 2008

Science du tourisme ou études touristiques ?

URI : https://id.erudit.org/iderudit/1070896ar

DOI : https://doi.org/10.7202/1070896ar

Aller au sommaire du numéro

Éditeur(s)

Université du Québec à Montréal

ISSN

0712-8657 (imprimé)

1923-2705 (numérique)

Découvrir la revue

Citer cet article

Hillali, M. (2008). La science du tourisme dans le discours des acteurs

internationaux : débat ou polémique ? Téoros, 27(1), 42-50.

https://doi.org/10.7202/1070896ar d'utilisation que vous pouvez consulter en ligne.

https://apropos.erudit.org/fr/usagers/politique-dutilisation/ 


\section{La science du tourisme dans le discours des acteurs internationaux Débat ou polémique?}

\section{Mimoun Hillali}

De nos jours, le tourisme est propulsé par son dynamisme social et économique au premier rang de l'économie mondiale. Cet essor sans précédent concerne tous les continents, dans des proportions inégales, mais tangibles. C'est pourquoi tout le monde en parle, en bien (intérêts évidents) ou en mal (inconvénients particuliers). Emblème de liberté, du libéralisme et même de fierté sociétale, le tourisme ne cesse de gagner en notoriété. II faut dire que les sociétés transnationales et les entreprises nationales touristiques, animées par des professionnels actifs, en tirent des profits juteux, malgré les incertitudes que fait planer le terrorisme sur le secteur. À côté de ces grands détenteurs de capitaux, une multitude de petites et moyennes entreprises, d'intermédiaires, de spéculateurs et de travailleurs en tirent directement ou indirectement, et à des degrés divers, quelque profit substantiel.

Mais le tourisme ne fait pas que des heureux; il irrite aussi un large éventail de populations, essentiellement les militants des valeurs éthiques, pour des considérations sociales, culturelles ou environnementales. Dans tous les cas, il ne laisse personne indifférent.

Aujourd'hui, les vacances, ayant atteint un stade de plein développement, subissent directement les évolutions générales de la société : chômage, inquiétude du lendemain, difficile entrée des jeunes dans la société, croissance des retraités " actifs » et à bon pouvoir d'achat, allongement de la vie, rejet des formes d'urbanisme des années soixante, évolution du parc automobile, développement des autoroutes, modification des coûts du transport aérien, rôle croissant d'Internet

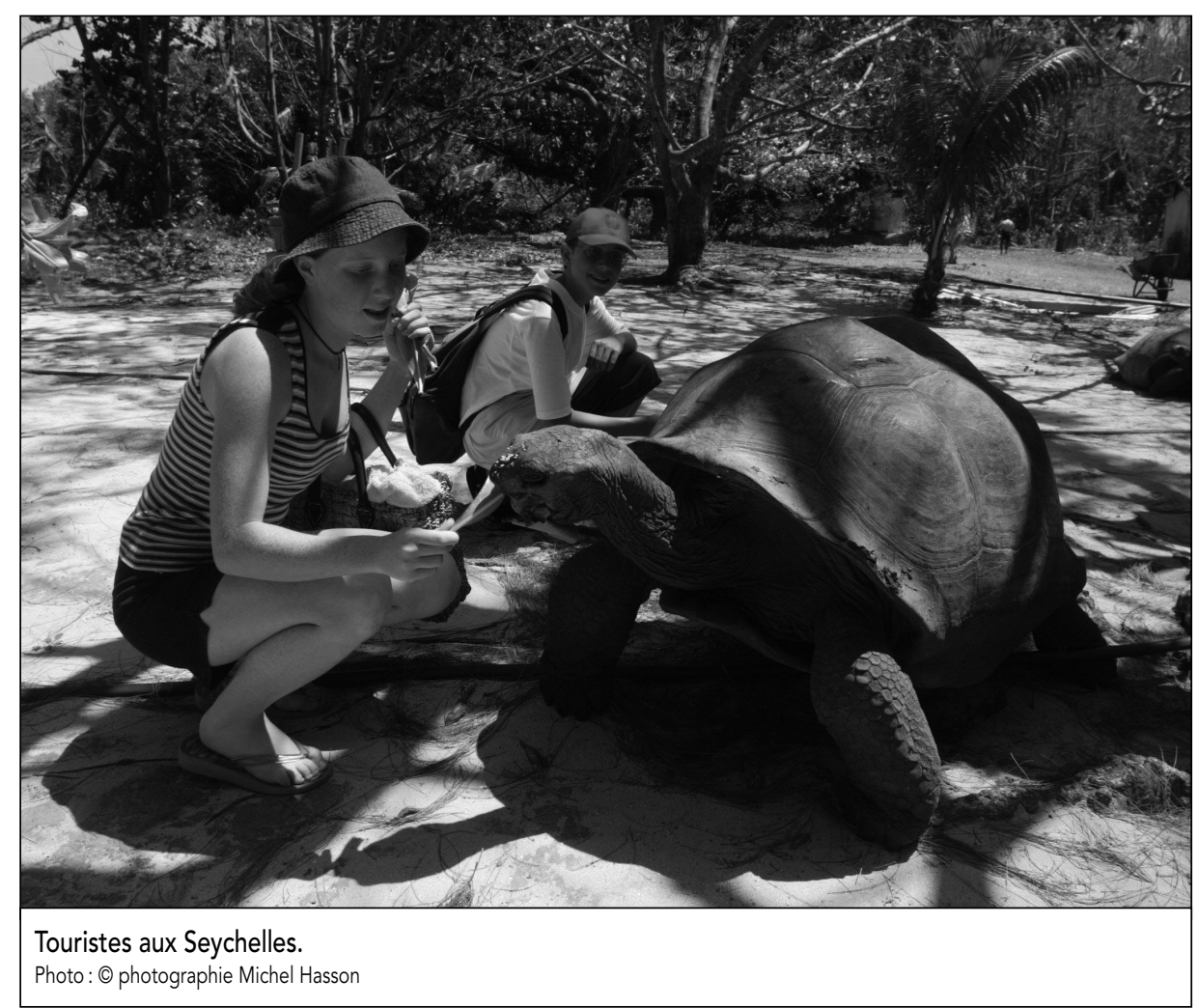

et de la société virtuelle, fusion progressive des temps et des espaces, réactions identitaires... (Viard, 2000 : 122)

Toutefois, plus le tourisme se porte bien, plus ses relations sociales, géopolitiques et économiques interfèrent et se compliquent en le projetant au devant de la scène internationale.

Et l'on comprend que le tourisme «se parle" dans toutes les langues et fait parler de lui dans toutes les sociétés. Mieux encore, il est enseigné dans la presque totalité des pays du monde et touche un grand nombre de sciences, de disciplines et de branches, allant des sciences sociales et économiques aux sciences juridiques et techniques. II est présent à tous les niveaux de l'enseignement, du secondaire au supérieur, de la maîtrise professionnelle à la formation continue...

Ce géant des voyages, des loisirs et de l'hôtellerie est accrédité par son triomphe d'un laissez-passer transcontinental, agréé par la mondialisation, surtout depuis la chute du mur de Berlin (1989). II ignore résolument les frontières, y compris celles où les lignes rouges de l'époque stalinienne ont juste viré à l'orange.

Jusque-là, tout va pour le mieux dans le meilleur des mondes de la mobilité d'agrément. Risquons tout de même un coup 
d'œil sur l'autre face de la médaille : ce secteur qui trône en tête de l'économie mondiale demeure, sur le plan scientifique, un gentil et aimable apatride. Et l'on constate que, en somme, ce colosse, fort impressionnant par son expansion économique et géographique, traverse une crise d'identité ou plutôt de filiation.

II faut préciser que les positions des adversaires et des partisans de la science du tourisme relèvent a priori de convictions personnelles. Certes, derrière chaque tribune animée par des penseurs de haut niveau se cache bien une foule d'intérêts, d'inquiétudes et d'espoirs aussi.

Le contexte est délicat, sans toutefois constituer une menace pour la grandeur d'un secteur en plein essor. Au contraire, disent les observateurs avertis, cette crise passagère est salutaire. Et d'expliquer qu'en atteignant les limites de l'impasse, la controverse s'achemine vers sa fin : ce sera alors le dénouement par concertation ou l'implosion pour mésentente. Dans un cas comme dans l'autre, le tourisme n'a plus rien à perdre.

En attendant le dénouement, le débat continue.

\section{"Bloc d'autonomie " et "front de refus ": une adversité amicale pour la bonne cause?}

Symbole de la mobilité d'agrément, de la sociabilité ludique, de l'employabilité accessible et de la rentabilité économique, l'activité touristique s'apprivoise et apprivoise aisément les milieux émetteurs en acclimatant les lieux d'accueil. Par quelque virtuosité, elle excelle à tisser de grands réseaux de production (espaces et produits), de distribution (négoces et intermédiations) et de communication (accueil et animation). Et, comme par miracle, les divergences politiques, les inégalités sociales et les différences culturelles des pays concernés semblent s'incliner devant «Sa Majesté » le tourisme.

Mais, signe des temps ou preuve d'une réussite inachevée, c'est au sein de la communauté des intellectuels et des chercheurs experts en la matière que le tourisme subit des revers inopinés dans son élan vers le couronnement de son succès en science loyalement reconnue!
Pour le moment, la question (science ou pas ?) n'en finit pas de faire des vagues. Deux types d'arguments plaident respectivement pour l'émancipation ou le statu quo.

II s'agit en pratique de deux courants de pensée nettement codifiés où s'expriment des sensibilités diverses. Conflit d'intérêts ou débat d'idées ou les deux à la fois, la réponse importe peu du moment que la réflexion anime une discussion ouverte qui éclaire l'opinion en braquant ses projecteurs sur un thème d'une grande actualité. Car, "sur ce thème de la science du tourisme, on peut rester divisé : c'est même un enrichissement » (Hoerner, 2000 : 18-20). Le ton se corse par moments chez les adversaires de la tourismologie, mais la tendance générale est à l'apaisement. «Travailler inlassablement à la légitimation sociale et scientifique de ce domaine, à une prise en considération équitable de son importance, est encore et toujours une priorité » (Cazes et al., 2001: 16-19) Pour la convenance d'usage, les deux protagonistes sont d'accord pour continuer à dialoguer; le front de refus " souhaite que le débat continue et s'élargisse... » (Cazes et al., 2001 : 16-19).

Les partisans de l'émancipation du tourisme, probablement plus nombreux, militent en faveur de sa reconnaissance universelle en tant que science à part entière. "Le tourisme cumule aujourd'hui un niveau de connaissances tel qu'il devient nécessaire de reconnaître à ses savoirs une autonomie et un champ disciplinaire spécifiques, indépendants des sciences dont ils sont originellement issus et dont ils conservent certaines méthodes. " (Vicériat et al., 2005 : 14-15)

Face à ces autonomistes convaincus, les gardiens du temple se raidissent sans toutefois rejeter l'idée d'une autonomie ultérieure du tourisme. Une bonne partie de ces doctes, et des meilleurs, pensent que, sans l'aptitude valorisante des disciplines reconnues scientifiquement et officiellement, le tourisme, jusque-là bénéficiaire de leur érudition, serait en danger! IIs prétendent que l'activité touristique, vue sous l'angle de la faisabilité scientifique, n'a pas encore atteint la maturité requise pour muer en science. « À force de vouloir hâter un accouchement, ne risque-t-on pas de donner naissance à un enfant handicapé ? " (Cazes et al., 2001: 16-19) Mais, semblent dire leurs adversaires, cet enfant est déjà né et se porte bien.
C'est plutôt son existence qui pose problème : ayant atteint la majorité, la question consiste à le doter d'une identité propre.

D'autres chercheurs paraissent se délecter de cette dualité gratifiante où la recherche touristique joue les "maitresses » attitrées. Sans refuser le port de la double casquette et sans se montrer corporatistes, ils affichent leur fierté d'être géographe, sociologue, économiste ou anthropologue, avec en plus le titre honorifique de spécialiste du tourisme. En s'appuyant sur la renommée des sciences-mères, nourricières de leurs savoirs de base et délégataires de titres scientifiques honorables, ils se targuent d'avoir une identité professionnelle originelle respectable! Ce dualisme n'est pas du goût des autonomistes. "lls ressemblent à ces 'étonnants voyageurs' qui croient échapper au phénomène touristique sous prétexte d'authenticité. » (Hoerner, 2000 : 18-20)

Quelques érudits humbles produisent, eux, discrètement. Pour ces «taupes" de la recherche touristique, les fondements scientifiques du tourisme priment sur ses rendements matériels. Ont-ils raison de rester dans l'ombre tout en se lançant sous les projecteurs de la notoriété par des publications qualitatives? «En résumé, beaucoup de zones d'ombre, de profondes évolutions, mais aussi l'extrême dispersion d'une foule d'acteurs plus ou moins solitaires " persistent (Lozes, 2000 : 54-58).

Une autre catégorie, qui se veut a priori neutre, publie occasionnellement quelques articles ou entreprend quelque étude ponctuelle sur le tourisme, assiste en spectateurs intéressés aux débats actuels. La démarche est d'autant plausible et possible que le tourisme, sorte de chasse non gardée, offre des occasions de dépaysement scientifique ou d'exercice didactique qui n'exigent ni engagement ni constance. II faut dire que le centre d'intérêt de ces excursionnistes ou saisonniers de la recherche touristique est ailleurs.

Ces remarques sont à présent dépassées. La bataille, toute la bataille, consiste aujourd'hui à en légitimer la reconnaissance en tant que science, et non à brandir une quelconque considération encenseuse de sa rentabilité ; autrement dit, il est beaucoup plus question à présent de son identité (propriété ontologique) que de sa capacité (considération axiologique). 


\section{Réussite socioéconomique et crise d'identité : l'envers du décor}

Le tourisme, grand générateur de revenus et d'emplois, attire une multitude de doctorants, d'étudiants, d'apprenants et de stagiaires. II faut dire que le chômage qui frappe les diplômés de certaines sciences sociales apporte de "l'eau bénite» au moulin du tourisme. Cette ruée vers les études touristiques et hôtelières a nécessité l'ouverture de nouvelles unités et branches de formation, ainsi que le recrutement de nombreux chercheurs, enseignants et formateurs. Évidemment, faute d'alternative, les contingents nécessaires à l'enseignement et à la recherche sont fournis par les sciences classiques. "Le tourisme compte de plus en plus de cadres de haut niveau, dont les compétences sont très mal reconnues. Naturellement, ils ont imputé cette lacune à l'absence d'une référence scientifique clairement définie. " (Hoerner, 2000 : 18-20) Et si c'était une façon détournée de limiter l'entrée au temple? "Dans ce sens, les géographes, les sociologues, les historiens, les économistes, les spécialistes de gestion, les juristes, les linguistes peutêtre, etc., qui s'intéressent au tourisme le tiennent jalousement en lisière. " (Hoerner, 2000: 18-20) Contrairement à la crainte émise par certains, la science du tourisme, une fois dotée de l'autonomie nécessaire, risque de bouleverser l'ordre préétabli des disciplines classiques (émigration des chercheurs et des formateurs, multiplication d'experts et de consultants, afflux d'étudiants et de doctorants).

Car, à tout hasard, la reconnaissance de la science du tourisme, à l'image de son avènement de masse, signifierait sa deuxième démocratisation. Les militants de l'autonomie du tourisme pensent que la réalité saute aux yeux, mais que, comme toutes les réalités éclatantes, elle fait détourner les regards de la nitescence que procure le changement vers la clarté singulière et rassurante de l'ordinaire.

II faut rappeler que les adversaires de la tourismologie sont composés de spécialistes et d'experts en tourisme de renommée internationale. Leur point de vue, habilement nuancé, laisse entendre que l'annexion actuelle du tourisme par les disciplines adoptives constitue une garantie avérée pour sa fiabilité et sa viabilité.
Comment ne pas percevoir, alors, les risques de fragmentation (certains observateurs parleront même de balkanisation) d'une telle aventure autonomiste, accompagnée d'une occultation soupçonneuse des disciplines encadrantes de cloisonnement, d'enfermement, d'insularisation thématique, le tout conduisant inévitablement à un surcroît de condescendance, d'ironie et de méfiance parmi les autres chercheurs, solidement adossés à leurs constructions scientifiques puissantes et bien identifiées. (Cazes et al., 2001 : 18)

À ce propos, la messe est dite; pourtant, il s'agit d'une naissance!

D'abord, si fragmentation ou balkanisation il y a, répliquent les autonomistes, elle concerne bel et bien la situation actuelle : le tourisme est fractionné ou morcelé entre différentes sciences ou disciplines ${ }^{1}$.

Les autonomistes pensent que c'est la situation actuelle qui empêche l'émergence de l'unité des acteurs et des actions, des méthodes et des programmes du tourisme appelés à se regrouper sous la coupole d'une science fédératrice. Cette dernière constitue la voie tout indiquée pour faire l'unité des actions et l'union des acteurs. Et même si le tourisme devait imploser, eh bien, qu'il le fasse en paix dans sa "propre demeure scientifique » et non en «réfugié » ou en «pensionnaire » discipliné. Et l'on comprend que « la recherche empirique en tourisme n'a pas encore conquis ses lettres de noblesse dans la confrérie sélecte des sciences » (Stafford, 1999 : 1). Communauté sélecte ou congrégation de sectes, la question n'est pas là. Que la requête des autonomistes n'aboutisse pas, cela ne prouve nullement que le tourisme soit encore mineur. II y a lieu de penser qu'il représente à présent un héritage important et que son éventuelle autonomie occasionnerait un manque à gagner pour certaines disciplines. L'hypothèse est à prendre au sérieux, lorsque, en plus, on prend en compte le nombre d'étudiants qui évitent actuellement de s'engager dans le tourisme, sorte d'impasse par manque de statut. Conséquemment, il est temps de clarifier les positions pour avancer en ayant de la visibilité.

Quant à l'ironie évoquée par certains auteurs, elle fait partie des critiques chroniques habituelles. « II existe une tradition fortement ancrée de dénonciation du tourisme internatio- nal de masse (Boorstin, 124-179). Ce qui est vilipendé en réalité n'est pas le tourisme en tant que tel, mais bien sa démocratisation. " (Amirou, 2000: 15-28) De plus, elle renvoie au passé (années 1950-1970), où l'enseignement et la recherche en tourisme n'en étaient qu'à leur début et n'attiraient pas grand monde.

Si le tourisme, en tant que sujet d'étude, a été longtemps considéré par les géographes français comme un sujet « frivole », ne suscitant que réserve et méfiance, il a été pour nous, encore plus difficile de faire admettre l'utilité de ce thème au sein de la communauté géographique marocaine. (Berriane, 1992 : 11)

Cette «tourismophobie » des années 1960 s'explique par une multitude d'équivoques. À l'époque, années du marxisme flamboyant au Maghreb, le tourisme était encore récent et même naissant dans certaines contrées. Et comme tout ce qui est nouveau et qui ne cadre pas avec le militantisme alimenté par le progressisme, tel qu'il a été admis à l'époque, le tourisme était taxé de néocolonialisme. Mais il semble que cette réticence touchait aussi les pays développés à la même époque.

Les implications spatiales des phénomènes liés au tourisme sont si considérables et évidentes qu'on est surpris des réticences, voire du dédain, longtemps manifestés par les géographes. Nombreux, et des meilleurs, étaient encore dans les années 1960 ceux qui tiennent le tourisme pour un thème mineur, presque marginal, de la recherche géographique. (Barbaza, 1966)

Les pionniers de la recherche touristique ont affronté à l'époque, avec courage et sérénité, une sorte de dénigrement alimenté à la fois par un conservatisme archaïque et un marxisme factice que l'effondrement du mur de Berlin a entraînés dans sa chute.

La recherche scientifique a très faiblement investi un champ pourtant très riche: rares sont les chercheurs qui consacrent, de façon continue et sur une longue période, l'essentiel ou une large part de leurs investigations aux aspects géographiques, entendez aux implications spatiales de manifestations économiques, sociales et environnementales, d'un secteur complexe dont la dynamique n'a cessé de s'affirmer. (Miossec, 1995 : 97-114) 
Aujourd'hui, les adeptes de la science du tourisme pensent que l'époque des ironies et des dérisions disculpées par la guerre froide est révolue. Certes, quelques traces subsistent encore.

La recherche en tourisme souffre déjà d'un déficit de considération scientifique: qu'en serait-il d'une hypothétique tourismologie refermée sur ses exigences professionnelles et sur sa scientificité autoproclamée, flatteuse sans doute pour les hyperspécialistes, mais aux effets redoutables dans la communauté scientifique. (Cazes et al., 2001: 16-19)

Mais imaginons pour la circonstance que la recherche en tourisme continue à faire de nos jours l'objet d'une quelconque ironie! Dans ce cas, une terrible question surgit et pose problème : les émérites venus d'autres sciences ont-ils aujourd'hui « honte » d'arborer leur titre d'expert ou de spécialiste en tourisme?

Bien au contraire, c'est plutôt l'inverse qui se remarque dans les faits : l'expertise en tourisme est devenue source de fierté, que ce soit pour le chercheur universitaire ou le praticien professionnel. C'est la vérité, n'en déplaise à ceux qui continuent à croire que le fait d'être bivalent constitue la preuve de virilité scientifique ou qui brandissent la double appartenance pour exorciser tout risque d'ironie!

Aussi, le temps n'est-il pas venu pour les sciences sociales et économiques d'admettre le fait accompli et de cesser de jouer les mères poules ? La relève des générations, y compris dans les sciences, fait partie des lois de la nature. Cela établi, comment expliquer - sans tomber dans la contradiction - que des enseignes de renommée reconnaissent au tourisme, au sein des universités du monde entier, le droit et la compétence d'exercer en lui réservant ordinairement un département sous contrôle d'une science «marraine »? Ce statut de franchise, à défaut d'un acte d'affranchissement officiel, bouleverse la donne: le tourisme devient science par contumace!

C'est l'ensemble de ces constats et de ces faits, qui accordent au tourisme un statut de réfugié de luxe, qui irrite et rassure à la fois les défenseurs de la science du tourisme.

Cela est dû, en partie, à l'absence de reconnaissance du tourisme comme matière d'enseignement, et de recherche «noble», des chercheurs se sont long- temps demandé si le tourisme pouvait être considéré comme un sujet de recherche digne des sciences sociales. Mentionnons aussi l'absence de supports universitaires, de publications, tel que cela se vit en Amérique du Nord avec, entre autres, les revues Annals of Tourism Research - il en existe d'autres, bien sûr - et Téoros pour les Québécois. Ajoutons que cette non-reconnaissance est aussi le résultat d'un émiettement des recherches entre géographie, économie, écologie, ethnologie, psychologie, histoire, communication, etc. Cela se soldant quelquefois par des querelles de légitimité disciplinaire par rapport à l'objet. (Amirou, 2000 : 29-53)

Pour les réticents, le tourisme, tout comme la recherche qui l'alimente, se porte bien dans sa position actuelle. Dans certaines mesures ils pensent qu'il est chanceux de pouvoir nourrir des recherches indéniables en se nourrissant à son tour des expériences confiantes des sciences aux méthodes de recherche et d'enseignement bien rôdées. En réalité, cet argument est à double tranchant: il sous-entend que le tourisme gagnerait à reprendre à son compte les outils et les méthodes que lui appliquent les sciences «parraineuses ", au point d'en faire -à leur insu? - une discipline autonome de fait ! Et l'on croit savoir que la science du tourisme s'impose en pratique, mais que l'étincelle autonomiste tarde à jaillir.

Est-il donc besoin de rappeler que, au stade où en est le développement du tourisme, scientifiquement parlant (enseignement, recherche, pédagogie, savoirs...), celui-ci est prêt à voler de ses propres ailes ? Au grand dam du rigorisme pragmatique ou dogmatique, souvent affilié politiquement, scientifiquement ou économiquement, l'avenir de la science du tourisme est assuré.

\section{Divergence théorique, convergence pratique : la bataille du tourisme n'aura pas lieu}

Le mouvement touristique est un phénomène structurel et structurant de grande envergure. II s'exprime mondialement par des déplacements, synonymes de défoulement de foule dans un élan de gaieté. II provoque, bon an mal an, des exodes périodiques ou saisonniers de plus en plus fréquents, occasionnant des recettes et des revenus impressionnants.
L'Organisation mondiale du tourisme (OMT) prévoit, à l'horizon 2020, un gigantesque mouvement touristique international de l'ordre de 1,6 milliard d'arrivées pour des recettes estimées à près de 2000 milliards de dollars. Une chose est sûre, le profit ira à la richesse dans le sens Nord-Nord et le Sud n'en récoltera que des miettes, en raison des contextes socioéconomique et politique qui y prédominent ; cette partie du monde ne donne, à l'exception d'un petit nombre de pays, aucun signe concret de changement. Mais, au-delà des chiffres, le tourisme remue le monde de fond en comble. C'est pourquoi toutes les sciences s'y intéressent.

Parler du tourisme du point de vue économique et social seulement, c'est oublier la valeur intrinsèque du phénomène et son essence particulière qui, au contraire, peut être mise en évidence dans une expression puriste assez significative qui, pénétrant dans le caractère le plus intime du phénomène, le caractérise comme " une conséquence naturelle de l'incarnation, un signe des temps, un instrument de dialogue. (lacono, 1971: 3)

Le tourisme est-il une victime, surtout de ceux qui sont censés le maîtriser pour le vulgariser et le mettre à la portée des masses ? Devrait-il incarner à jamais cette image équivoque et indécise de pause récréative destinée à rompre la routine du labeur quotidien en offrant aux sciences sociales bien établies l'occasion de venir s'y exercer avec talent? Faudra-t-il penser, en jouant de la psychologie élémentaire, que le tourisme est mal aimé (domaine de permissivité), mais très fortement désiré (activité fructueuse) ? "On a souvent associé le tourisme aux quatre ' $S$ ' pour 'sea/sun/sand/sex'. En fait, la réalité est plus complexe et plus diversifiée. Le présent essai explore une autre facette de la pratique touristique, celle du cinquième ' $S$ ' en référence au tandem science/technologie." (Bédard, 1996 : 2) Et des S, il y en a encore et il en y aura toujours tant que le tourisme est en pleine croissance: "survection ${ }^{2}$ ", sécurité, saturation, surbooking...

Le tourisme et le touriste ont toujours fait l'objet d'une perception réductrice. Dans les décennies 1950-1970, les écrits se concentrent sur le jugement et les critiques envers le tourisme et le touriste [....] sur fond d'analyse idéologique de la société. Si l'on assiste à une reconnaissance du tourisme dans les années 1980-1990, 
l'approche est alors centrée sur une réhabilitation du sens de l'expérience du touriste face au mythe et au modèle du voyageur. (Kadri et Bédard, 2006 : 62-64)

Décidément, malgré son couronnement socioéconomique notoire, le tourisme demeure prisonnier des visions où se mêlent méfiance et vigilance précautionneuse. Celles-ci contribuent à son cantonnement, sur les plans institutionnel et conventionnel, en le confinant dans un statut de branche universitaire mineure, malgré sa notoriété! Autant dire que le tourisme est maître incontesté dans le domaine socioéconomique, mais pas de son propre destin scientifique.

Et c'est avec raison que d'autres chercheurs ont mis l'accent sur "l'importance d'asseoir la construction des connaissances sur une vision de globalité et de complexité du tourisme " (Kadri et Bédard, 2005 : 77-80; Kadri et al., 2005 : 62-64).

II n'est pas rare de voir des chercheurs érudits faire l'éloge de la science du tourisme tout en hésitant à opter pour son autonomie. "Que les études en tourisme souffrent d'une image non valorisante dont les conséquences sont désastreuses en termes de crédit pour les chercheurs, en termes de notoriété pour les professeurs et en termes de débouchés pour les étudiants, nul aujourd'hui ne le nie. » (Bessières, 2004 : 1)

Mais faut-il pour autant créer et promouvoir un nouveau mot : « tourismologie », avec pour conséquence pratiquement inévitable de qualifier les chercheurs et les enseignants qui l'étudient de "tourismologues » et ses détracteurs de «tourismopathes »? Ce n'est pas certain. Le terme « tourismologie ", même s'il correspond bien étymologiquement au "discours" sur le tourisme, véhicule une image savante, précieuse voire prétentieuse. Elle évoque une spécialité unique alors que sa spécificité est d'être multidisciplinaire, au confluent des sciences économiques et des sciences humaines. Restons humbles. (Bessières, 2004 : 1)

\section{Le géant mondial des voyages et des loisirs est un " roi sans royaume "}

Quittons ce panthéon où les plaidoyers ont un caractère hautement intellectuel et scientifique et descendons sur le terrain du tourisme au quotidien. Car, en présence de ces mêlées de haute voltige où se côtoient probablement qualité d'analyse et délits d'initiés, circonstances atténuantes et règlements de compte, en plus d'une franchise objective ou subjective, le profane, lui, est quelque peu désorienté. Et pour cause! Le tourisme lui semble servir, tantôt de terrain de bataille pour initiés, tantôt de trophée de guerre pour savants. Dans ce cas, il est recommandé de coller à la réalité, en prospectant l'autre versant du secteur, celui du commun des mortels qui vit ou survit grâce au tourisme. Le tourisme, par sa diversité professionnelle et sa popularité sociale, constitue un filon exploitable qui procure emplois, revenus et recettes à des millions de personnes des cinq continents, en plus des plaisirs divers (d'autres diront pervers) qui font fructifier ces apports économiques et agrémentent ces rapports sociaux.

Tableau 1

Arrivées et recettes du tourisme international : 1990, 1995, 2000, 2005 et 2006

\begin{tabular}{|c|c|c|c|c|c|c|c|c|}
\hline Zone d'arrivées & 1990 & 1995 & 2000 & 2005 & 2006 & $\begin{array}{l}2006 / \\
2005\end{array}$ & $\begin{array}{l}1990 \\
\text { parts } \\
\text { (en \%) }\end{array}$ & $\begin{array}{c}2006 \\
\text { parts } \\
\text { (en \%) }\end{array}$ \\
\hline \multicolumn{9}{|l|}{ Europe } \\
\hline Arrivées & 265,6 & 315,0 & 392,5 & 438,3 & 456,9 & 4,2 & 60,4 & 54,3 \\
\hline Recettes & 142885 & 212159 & 232559 & 350545 & 377625 & 7,7 & 52,9 & 51,3 \\
\hline \multicolumn{9}{|l|}{ Afriques } \\
\hline Arrivées & 15,2 & 20,4 & 27,9 & 37,3 & 40,9 & 9,7 & 3,5 & 4,9 \\
\hline Recettes & 6402 & 8500 & 10404 & 21638 & 24237 & 12,0 & 2,4 & 3,3 \\
\hline \multicolumn{9}{|l|}{ Asie-Pacif. } \\
\hline Arrivées & 56,2 & 82,4 & 110,6 & 155,4 & 167,4 & 7,7 & 12,8 & 19,9 \\
\hline Recettes & 46474 & 80700 & 85222 & 144678 & 153461 & 14,1 & 17,2 & 20,9 \\
\hline \multicolumn{9}{|l|}{ Amériques } \\
\hline Arrivées & 92,8 & 109,0 & 128,2 & 133,2 & 136,0 & 2,1 & 21,1 & 16,2 \\
\hline Recettes & 69274 & 98439 & 130800 & 144678 & 153351 & 6,0 & 25,6 & 20,9 \\
\hline \multicolumn{9}{|l|}{ M.-Orient } \\
\hline Arrivées & 9,6 & 13,7 & 24,5 & 38,3 & 40,7 & 6,3 & 2,2 & 4,8 \\
\hline Recettes & 5124 & 10905 & 15242 & 26248 & 26757 & 1,9 & 1,9 & 3,6 \\
\hline \multicolumn{9}{|l|}{ Monde } \\
\hline Arrivées & 439,4 & 540,5 & 684,0 & 802,0 & 842,0 & 5,0 & 100 & 100 \\
\hline Recettes & 270159 & 410703 & 474227 & 677611 & 735441 & 8,5 & 100 & 100 \\
\hline
\end{tabular}

Source : OMT, 2007. Arrivées en millions de personnes et recettes en millions USD. 
À part les professionnels, les chercheurs, les écrivains et d'autres acteurs connus sur la scène touristique mondiale, l'opportunisme, le hasard ou la nécessité ont souvent mis sur la voie de l'industrie touristique des bénéficiaires de l'ombre, avec ou sans compétences requises, mais doués pour la débrouille. C'est ainsi que des opportunistes patentés et des profiteurs occasionnels n'hésitent pas à s'y aventurer par intérêt ou par nécessité. II est admis que, en dehors des professionnels compétents et intègres, des acteurs féaux et attentifs, des chercheurs aptes et expérimentés, le tourisme, en tant que gisement à ciel ouvert, donc d'accès facile, est abordable pour des cohortes d'amateurs fortunés, de parasites véreux et même d'usurpateurs d'identité. Ce beau mélange insondable où le gentleman en costume trois-pièces croise sans gêne le baba cool au piercing et au tatouage remarquables, donne lieu à un conglomérat socioprofessionnel hétérogène et composite où l'opportunisme des uns n'a d'égal que la loyauté des autres.

C'est à ce niveau que la nécessité absolue d'une science du tourisme se fait sentir. Ce caractère hétéroclite des acteurs et des activités, en plus de la pléthore des disciplines encadrantes, contribue à la multiplication des approches, des intérêts et des objectifs. En conséquence, le secteur n'est pas abordé de façon globale en tant que champ d'étude homogène, certes complexe, mais distinct. Tout le monde y trouve son compte, peut-être, sauf le tourisme. Celui-ci est étudié par branches d'activités, par tronçons d'espaces ou par périodes occasionnelles par des chercheurs qui le tiennent souvent pour un exercice ou un apport d'appoint. II en résulte que l'intérêt manifesté par tout ce monde demeure aléatoire du moment que l'apport et l'estime sont manifestement situés ailleurs : le tourisme n'est en fin de compte que le complément ou la valeur ajoutée d'une science-mère.

Une approche globale ne serait-elle pas nécessaire pour aider à segmenter techniquement et à baliser méthodiquement ce vaste champ, dans le souci de mettre en place les procédés fondateurs d'une science du tourisme?

Mais n'exagérons rien. La pluridisciplinarité, la transversalité et même l'intrusion sont, dans leur essence, salutaires ; elles tendent à rehausser la qualité de nombreuses études et recherches dédiées au tourisme dans un vaste concert de disciplines. La jonction entre la diversité des approches et le fruit des résultats dote les savoirs en tourisme d'une banque de données, certes dispersées, mais très appréciées. Cette allusion se réfère à l'existence d'un cadre disciplinaire doté de méthodes d'inspiration multidisciplinaire qui passent déjà pour propres à la recherche touristique (cas du compte satellite) ; éparpillées en diaspora pédagogique ou didactique, ces approches fonctionnelles sont prêtes à l'autonomie et à rejoindre une sorte de mère-patrie, le moment venu.

Toutefois, en cas de reconnaissance de la science du tourisme, il va falloir attendre un certain temps avant d'espérer voir les premiers diplômés se targuer d'être tourismologues ou téorologues avec satisfaction et fierté. Ce jour-là, la nouvelle vague de scientifiques pourra s'autoriser, à son tour, à effectuer des excursions de courtoisie dans les domaines de la géographie, de la sociologie, de l'économie... sous l'égide de la tourismologie géographique, sociologique ou économique. II n'y aurait rien d'étonnant à cela. Comme on parle de la géographie touristique ou de la sociologie des loisirs, la future science du tourisme aura tout le loisir d'aller explorer les domaines réservés des anciennes sciences tutrices. Et ce sera avec émotion et fierté que l'apprenti, devenu maître, libre et reconnu, entreprendra de revisiter les lieux initiateurs et promoteurs des fondements de ses savoirs.

Ce constat illustre bien l'importance des plaidoyers en faveur de prises de positions claires qui permettent de faire le point. Cela compte beaucoup pour les pays où les ingérences sont monnaie courante. De plus, ces débats ouverts et francs peuvent aider les sans opinion, sinon à prendre position en toute connaissance de cause, du moins à réagir et à enrichir un peu plus le socle d'une science en devenir. Les débats hautement intellectuels ont déblayé le terrain et posent, avec pertinence, les éléments fondamentaux de la problématique «pour ou contre » la science de tourisme. Le moment est capital pour son avenir, en attente du baptême universel d'une nouvelle science. «Ensemble pour la reconnaissance d'une science du tourisme » (Vicériat et al., 2005 : 14-15) est un appel solennel qui prouve la maturité des savoirs en tourisme, de l'avis des membres de l'AFEST'3 ${ }^{3}$ association qui a fêté en 2007 son $50^{\circ}$ anniversaire.
Aujourd'hui, plus que jamais, l'avenir scientifique du tourisme se pose en termes existentiels de type shakespearien : être ou ne pas être parmi les sciences reconnues en ce début du troisième millénaire.

\section{Aux origines d'une particularité circonstancielle : le tourisme est-il né " sous $x$ " ?}

Le tourisme, dans sa dimension scientifique (formations, études, recherches, savoirs, enseignements...), se fait héberger par les sciences prestigieuses; mais il lui arrive de faire des vagues, surtout depuis que son champ d'action s'élargit à vue d'œil et lui permet de gagner en considération et en prestige.

Le tourisme cumule aujourd'hui un niveau de connaissances tel qu'il devient nécessaire de reconnaître à ces savoirs une autonomie et un champ disciplinaire spécifique, indépendant des sciences dont ils sont originellement issus, et dont ils conservent certaines méthodes. (Vicériat et al., 2005 : 14-15)

Par ailleurs, le développement du tourisme est ancré dans les mœurs et les économies des pays développés et ne cesse de subir l'influence des nombreux bouleversements des sociétés postindustrielles, en y produisant à son tour sa propre influence. Ces interactions d'ordre socioéconomique s'inscrivent dans l'accélération et l'expansion de l'internationalisation des échanges. Le tourisme s'éveille et éveille des intérêts divergents. Dès le départ ce succès s'est accompagné de quelques couacs, comme l'a rappelé, à la fin du siècle dernier, un éminent spécialiste de la sociologie du tourisme en posant cette question quelque peu insolite : «L'histoire du touriste a-t-elle approfondi ce personnage [le touriste] qui, longtemps après sa naissance, n'avait pas encore de nom? A-t-elle examiné les contextes économiques, politiques ou sociaux de ses départs? De ses périples ? (Laplante, 1996: 10). Et pourtant, en tant que voyageur, le sujet a fini par avoir un nom : « touriste ", alors que le chercheur spécialiste ou expert en est encore à se demander : "Qui suis-je »? À propos, comment appelle-t-on un chercheur en tourisme? Car, et jusqu'à nouvel ordre, le chercheur en tourisme n'a pas encore de nom distinctif, ou plutôt d'identité professionnelle! Pourtant il anime des formations, des enseignements et des recherches qui font la gloire des 
sciences sociales ou économiques reconnues et qui lui offrent, en contrepartie, une sorte de satisfaction morale. Mais il n'existe pas non plus de nom pour qualifier ce noble domaine du chercheur (téorologie? tourismologie?...) qui nourrit ses observations et ses investigations. A-t-on mis la charrue avant les bœufs? «Si d'aventure, un universitaire était tenté par 'l'expérience touristique', il ne peut s'y adonner qu'une fois sa carrière bien positionnée, car en fait, si la noria n'est pas alimentée en eau vive, elle ne peut rester en mouvement. " (Pagnon-Maudet, 2000 : 65-66)

Alors, tout compte fait, quel mal y a-t-il à intervertir l'ordre des choses en parlant, à titre d'exemple, de tourismologie géographique ou de téorologie économique? Apparemment rien; à moins d'attacher une importance particulière aux fonctions au détriment des qualifications. C'est pourquoi il devient assez urgent d'aller au-delà des discours courants ou dominants à la recherche d'ententes adéquates, pour le plus grand bien du secteur et de ceux qui y travaillent.

Mais alors, disent les adeptes de la polyvalence, où est le problème? "La réunion de chercheurs expérimentés de multiples origines disciplinaires allait, fort opportunément, dans la direction que nous voulions privilégier, celle d'une interdisciplinarité effective, ouverte et constructive, valorisant les divers apports scientifiques encadrants. " (Cazes et al., 2001: 16-19)

On le sait bien, "la géographie, ça sert, d'abord, à faire la guerre » (Lacoste, 1976) et parfois à faire de la résistance aussi. Au siècle dernier, les géographes ont rejeté en bloc l'étude du tourisme par rigorisme scientifique. Et ils pensaient dur comme fer avoir raison!

Le tourisme, en tant qu'objet scientifique, a été tardivement abordé par les géographes [...] Car la diversification de l'objet scientifique de la géographie se heurte alors aux rigidités de l'orthodoxie disciplinaire. Les gardiens du temple délimitent strictement ce qui relève de la géographie (la géomorphologie, la géographie rurale et les paysages ruraux) et ce qui en est exclu. (Violier, 2000 : 17-20)

Et, comme par hasard, ce sont les géographes qui se montrent aujourd'hui les plus résistants face à l'autonomie du tourisme!

\section{Débat libre au Nord, calme plat au Sud? Les uns "parlementent ", les autres pas...}

Pour l'observateur des pays en développement (PVD), la controverse est surprenante: à en juger par certains témoignages, les pays développés semblent recourir à l'informel supérieur toléré, pour débloquer la situation en attendant l'officiel! «Que dire également des thèses ? J'en dirige un certain nombre que, faute de mieux, je range sous la rubrique Aménagement. » (Hoerner, 2000: 18-20) Mais n'est-ce pas là le signe irréfutable d'une démocratie qui donne à la concertation toutes les garanties de réussite?

Dans les PVD, tout porte à croire que la nouveauté, mère par essence de rivalités, a toujours constitué une menace pour «l'acquis incorporé » ou pour "l'ordinaire rassurant ». Ces États récusent toute innovation porteuse d'interférences supplétives ou compétitives. II est bien connu que la stagnation est défendue socialement, économiquement et politiquement au non d'une authenticité qui ne dit pas son nom. C'est ainsi que le monde avance et que l'Afrique n'est pas du voyage. Et ce n'est pas pour autant que l'authenticité en sort indemne!

Au Maghreb, par exemple, il existe une génération qui a formidablement profité de la période postcoloniale et, opportunément, de celle dite " post-soixante-huit » pour faire des études poussées, notamment en France. C'était une époque où les bourses et les visas n'étaient pas réservés à l'élite. En revenant dans leur pays d'origine, ces chanceux ont profité de circonstances particulièrement favorables. Ils ont pris la relève des Européens retournés chez eux après l'indépendance - au moment où le tourisme connaissait un démarrage, certes timide, mais constant - pour animer la recherche, la publication et l'enseignement.

Ces chercheurs issus des pays en développement, mais formés en Occident, semblent

devoir, de façon presque inéluctable, adhérer par conviction ou s'affilier par nécessité, ou, tout au moins s'inspirer par accommodation de l'une de ces écoles de référence. Et l'on peut se demander s'il existe vraiment d'autres approches ou façons de procéder pour un chercheur du Sud, en dehors des méthodes d'études et des méthodologies d'analyse des cou- rants et des styles élaborés et légués à la recherche en tourisme, par les pères fondateurs de ces écoles de renommée internationale. La réponse est oui et non [...]

- Oui, si le chercheur originaire du tiersmonde, travaillant dans un contexte national ou régional, se décide à s'impliquer par le biais de rôles initiatiques, lors de ses investigations; en se faisant simultanément et successivement « expert, citoyen et objet » de sa recherche, il pourra aboutir à des résultats remarqués [...]

- Non, si le chercheur part déjà convaincu que sa démarche, scientifiquement établie, et sa mission, rationnellement mûrie, sont inévitablement bonnes à révéler la vérité, toute la vérité, sur le thème de sa recherche. (Hillali, 2003 : 4)

C'est avec intérêt et agrément que l'autonomie du tourisme arrive à point nommé pour faire profiter la recherche touristique des approches certifiées conformes des autres sciences, mais sans la rendre prisonnière des idiomes fondateurs ni des procédés initiateurs. Car le problème majeur des PVD réside dans le décalage entre la rapidité de l'évolution des technologies (cas de l'informatique) et la lenteur d'assimilation des nouveautés par les populations en majorité analphabètes. Cela pose, une fois de plus, le problème de la technologie produite dans le Nord face à un Sud cantonné dans le rôle de consommateur, de surcroît pauvre. En plus de cette contrainte majeure, d'autres facteurs, surtout d'ordres socioculturel et politique, ne militent pas en faveur de l'actualisation prompte des innovations et des savoirs. Si, en outre, il faut s'attendre chaque fois que nécessaire à ce que la mise au point des sciences et des technologies vienne de l'extérieur, le développement n'est pas pour demain.

À cet égard, bien des universités des PVD ont déjà admis la science du tourisme dans leurs cursus ou corpus universitaires. "La science du tourisme est maintenant enseignée au Portugal, au Maroc, en Algérie, en Tunisie, au Liban, au Vietnam, à Madagascar, ainsi que dans plusieurs pays anglophones. " (Hoerner, 2007 : 74) Ignorant souvent l'existence de ce débat Nord-Nord ${ }^{4}$ sur la science touristique, ces pays ont commis l'initiative sans controverse ni polémique. Eh bien, pour une fois où les derniers de la classe créent la surprise, l'événement mérite 
d'être salué vivement par solidarité morale ou par simple soutien à l'innovation, si modeste soit-elle. La raison de cette reconnaissance est toute simple. En Afrique (faut-il le rappeler?), la majorité des sciences humaines modernes sont arrivées, à quelques exceptions près, dans les bagages des explorateurs ou des chercheurs coloniaux. "La géographie moderne au Maroc est fille de la colonisation. » (Naciri, 1984 : 310-343) C'est pourquoi la science du tourisme serait la bienvenue après toutes ces gestations et discussions libres. Pour preuve, elle s'épanouit mieux qu'une démocratie importée sur des chars.

En Afrique, le diplôme supérieur demeure la seule voie qui mène à l'émigration (fuite des cerveaux) ou à l'intégration sociale (emploi et reconnaissance), donc à l'ascension sociale. Le tourisme y offre aujourd'hui une belle palette d'emplois dans une vingtaine de pays. Et il faut rappeler que les postes-clés (direction, gestion...) des principales multinationales sont fréquemment occupés par des cadres étrangers.

\section{Conclusion}

Historiquement, les sciences humaines ont fait leur apparition et, en même temps, leur apprentissage, sur des terrains socioéconomiques et géopolitiques en friche. Le tourisme, lui, peut se targuer, avec raison, d'être la seule science qui a fait, un demi-siècle durant, des stages édifiants dans diverses sciences, en profitant de l'encadrement " des chercheurs solidement adossés à leurs constructions scientifiques puissantes et bien identifiées. " (Cazes et al., 2000: 16-19). Le fait qu'il soit parrainé et entrainé par plusieurs sciences est une particularité exceptionnelle qui prouve sa grande maturité et qui en rehausse le caractère scientifique. C'est ce qui fait dire aux défenseurs du tourisme "qu'il doit être circonscrit dans tous ses aspects, sans complaisance, et faire naître une réflexion critique qui empruntera beaucoup aux autres sciences humaines et sociales » (Hoerner, 2000 : 18-20).

La science du tourisme serait, une fois indépendante, la seule discipline récente à entrer de plain-pied dans le temple sacré avec un legs avéré et un curriculum vitae enviable; est-il nécessaire de rappeler qu'elle a reçu en héritage des enseignements, des méthodes et des approches des sciences sociales, économiques, juridiques et techniques?
Ces legs et ces échanges ont permis d'étoffer la discipline, en supervisant son initiation, au point de la munir d'atouts dont certaines sciences classiques ne peuvent plus se prévaloir. Et l'on comprend l'inquiétude des disciplines traditionnelles qui hébergent la recherche en tourisme en lui octroyant souvent un statut rentable de "sans-papiers ».

Connaître son identité disciplinaire et reconnaître son port d'attache scientifique, pour éviter des célébrations sous tutelle, ne peut que contribuer à l'épanouissement du chercheur et de la recherche en tourisme. L'égalité des chances et la légalité de l'acte sont les clés de sa réussite. Ensemble, tout devient possible. "Liés à nos frères par un but commun et qui se situe en dehors de nous, alors seulement nous respirons et l'expérience nous montre qu'aimer ce n'est point nous regarder l'un l'autre mais regarder ensemble dans la même direction. " (Saint-Exupéry, 1964 : 225) Et l'espoir nous anime et nous interpelle pour regarder dans la bonne direction : celle de l'avenir du tourisme.

Toutefois, il faut rendre hommage aux militants de la bonne heure et reconnaître à l'occasion que la problématique de base est digne de la polémique qui couve.

Mimoun Hillali est professeur à l'Institut supérieur international de tourisme de Tanger, Maroc.

\section{Notes}

1 Les principales sont: géographie, économie, gestion, marketing, droit, histoire, sociologie, anthropologie, ethnologie, psychologie, sciences politiques...

2 Terme d'Eiglier et Langeard, cités par Origet du Cluzeau (2000 : 115).

3 Association française des experts et scientifiques en tourisme, fondée en 1957.

4 Ces discussions prennent par moments des allures de débats franco-français ou francoquébécois.

\section{Bibliographie}

Amirou, Rachid (2000), "Éléments pour une sociologie qualitative du voyage et du tourisme", La recherche en tourisme, Actes du colloque de Foix, Paris, Éditions Jacques Lanore, p. 29-53.

Barbaza, Yvette (1966), Le paysage humain de la Costa Brava, Paris, Éditions Armand Colin.
Bédard, François (1993), La valorisation des sciences et de la technologie par le tourisme, Texte inédit.

Berriane, Mohamed (1991), Tourisme national et migrations de loisirs au Maroc, Rabat, Université Mohamed V; Publications de la faculté des Lettres et des sciences humaines, série « Thèses et mémoires ", nº 16.

Bessières, Jacques (2004), Non à la tourismologie, oui à l'ennoblissement du tourisme, [http://www.afest.org/article356.html].

Boorstin, Daniel (1971), Du voyage au tourisme: l'art oublié du voyage, L'image, Paris, UGE, coll. «10/20».

Cazes, Georges, Jean-Didier Urbain, Jean-Michel Dewailly, Phillipe Bachimon et Vincent Vlès (2001), « À propos du tourisme. Science par autoproclamation ? ", Revue Espaces, n० 178 , janvier, p. 17-19.

Eiglier, I.P. et E. Langeard (1987), La survection, le marketing des services, Mac Graw-Hill.

Hillali, Mimoun (2003), Le tourisme international vu du Sud, Essai sur la problématique du tourisme dans les pays en développement, Sainte-Foy, Québec, Presses de l'Université du Québec.

Hillali, Mimoun (2007), La politique touristique du Maroc, diagnostic, bilan et critique, Paris, Éditions l'Harmattan.

Hoerner, Jean-Michel (2000), Pour la reconnaissance d'une science touristique, Revue Espaces, no 173, juillet-août, p. 18-20.

Hoerner, Jean-Michel (2002), Traité de tourismologie : Pour une nouvelle science du tourisme, Perpignan, Presses universitaires de Perpignan, coll. «Études ».

Hoerner, Jean-Michel (2007), Géopolitique du tourisme, Paris, Armand Colin.

lacono, A.Q. (1971), "Propos historiques sur le tourisme ", Les cahiers du tourisme, série B, $n^{\circ} 12$, Centre d'études du tourisme, Aix-enProvence, p. 3.

Kadri, Boualem et François Bédard (2006), « Vers les «sciences du tourisme " ? Complexité et transdisciplinarité ", Téoros, vol. 25, n 1 , p. 77-80.

Kadri, Boualem, Myrabelle Chicoine et François Bédard (2005), "Vers une science du tourisme; La contribution des revues Téoros et Loisir et Société", Téoros, vol. 24, n³, p. 61-64.

Lacoste, Yves (1988) [1976], La géographie, ça sert, d'abord, à faire la guerre, Paris, La Découverte.

Laplante, Marc (1996), L'expérience touristique contemporaine: fondements sociaux et culturels, Sainte-Foy, Québec, Presses de I'Université du Québec, coll. "Tourisme ».

Lozes, Jean (2000), Approches collectives et individuelles : diversité et enrichissement, La recherche en tourisme, Actes du colloque de 
Foix, Paris, Éditions Jacques Lanore, p. 54-58.

Miossec, Jean-Marie (1995), "Tourisme dans le monde arabe: Méthodes et concepts", Recherches urbaines dans le monde arabe, $n^{\circ} 24$, p. 97-114.

Naciri, Mohamed (1984), « Perception de l'espace et des lieux; la géographie coloniale : une science appliquée à la colonisation ", Connaissances du Maghreb (sciences sociales et colonisation), Éditions du CNRS, coll. "Recherches sur les sociétés méditerranéennes ", p. 310-343.
Origet du Cluzeau, Claude (2000), Le tourisme culturel, Paris, Presses universitaires de France, coll. «Que sais-je ? ».

Pagnon-Maudet, Christine (2001), « La recherche en tourisme et hôtellerie: blocage et factures d'évolution en France", La recherche en tourisme, Actes du colloque de Foix, Paris, Éditions Jacques Lanore, p. 65-66.

Saint-Exupéry (de), Antoine (1964), Terre des hommes, Paris, Gallimard.

Stafford, Jean (1999), La recherche touristique. Introduction à la recherche quantitative par questionnaire, Sainte-Foy, Presses de I'Université du Québec.

Viard, Jean (2000), Court traité sur les vacances, les voyages et l'hospitalité des lieux, Paris, Éditions de l'Aube.

Vicériat, Patrick, Claude Origet du Cluzeau et Michel Balfet (2005), « Ensemble pour la reconnaissance d'une science du tourisme", Revue Espaces, no 224, p. 14-15.

Violier, Philippe (2000), « Fin de siècle : la géographie du tourisme à la croisée des chemins ", La recherche scientifique en tourisme, Actes du colloque de Foix, Paris, Éditions Jacques Lanore, p. 17-20.

\section{TÉQROS \\ 1) Revue de recherche en tourisme}

\section{Appel à textes}

En dehors des dossiers thématiques TÉOROS ouvre ses pages aux propositions spontanées. La rédaction invite tous les chercheurs qui oeuvrent dans le domaine du tourisme ou qui s'intéressent au tourisme sous tous ses aspects, à soumettre des articles de nature analytique à la revue. On peut soumettre un article en l'envoyant à :

\section{teoros@uqam.ca}

Publiée trois fois I'an depuis 1982, la revue Téoros de recherche en tourisme s'affirme dans la francophonie comme le leader de diffusion des résultats de recherche et de réflexions fondamentales et appliquées dans une perspective multidisciplinaire. Les problématiques liées aux rapports entre le tourisme et la culture ou entre le tourisme et la société, à la gestion ou à la planification en tourisme, à des cas particuliers de mise en tourisme ainsi qu'aux questions méthodologiques d'actualité, par exemple, comptent parmi les sujets abordés.

Les textes soumis doivent apporter une contribution scientifique originale, que ce soit par le biais d'information factuelle, jusqu'alors inconnue ou par une nouvelle interprétation d'un thème particulier. Téoros vise avant tout le transfert de connaissances; son objectif est donc de promouvoir une meilleure compréhension des phénomènes liés au tourisme.

Les auteurs doivent faire parvenir un manuscrit présenté selon les règles de la revue, disponibles au www.teoros.uqam.ca.
Habituellement, un article analytique compte environ 6000 mots et n'excède pas 8000 mots avec trois ou quatre illustrations. On pourra cependant considérer des textes plus longs ou plus courts. Les articles peuvent être soumis en anglais ou en français et doivent être accompagnés d'un résumé de 100 à 200 mots (français/anglais) et de cinq mots-clés.

La publication des articles se fait sous réserve d'une évaluation. Tous les manuscrits seront évalués anonymement par des pairs qui pourront faire des suggestions ou demander des modifications. La rédaction transmettra l'avis des évaluateurs aux auteurs et s'assurera que les modifications demandées seront apportées.

Au plaisir de vous lire dans nos pages. 